\title{
Regulation of chondrogenesis and chondrocyte differentiation by stress
}

\author{
Michael J. Zuscik, Matthew J. Hilton, Xinping Zhang, Di Chen, and Regis J. O’Keefe
}

Center for Musculoskeletal Research, University of Rochester Medical Center, Rochester, New York, USA.

\begin{abstract}
Chondrogenesis and endochondral ossification are the cartilage differentiation processes that lead to skeletal formation and growth in the developing vertebrate as well as skeletal repair in the adult. The exquisite regulation of these processes, both in normal development and in pathologic situations, is impacted by a number of different types of stress. These include normal stressors such as mechanical loading and hypoxia as well pathologic stressors such as injury and/or inflammation and environmental toxins. This article provides an overview of the processes of chondrogenesis and endochondral ossification and their control at the molecular level. A summary of the influence of the most well-understood normal and pathologic stressors on the differentiation program is also presented.
\end{abstract}

\section{Introduction to cartilage}

Cartilage is a connective tissue that is comprised primarily of matrix (mainly collagens and proteoglycans) containing relatively sparse populations of chondrocytes, which perform matrix-generation and maintenance functions. During the development and growth of vertebrates, chondrogenesis is the dynamic cellular process that leads to the establishment of various types of cartilage, including hyaline, fibrous, and elastic cartilage. Hyaline cartilage is found in craniofacial structures, the trachea and bronchial tubes, the articular surfaces of diarthrodial joints, and the growth plate (GP) of long bones. GPs are responsible for driving the process of limb lengthening and bone growth during development pre- and postnatally. This type of bone growth involves the process of endochondral ossification (otherwise known as bone formation). The type of cartilage that is most prominent and most susceptible to both normal and pathologic forms of stress is the hyaline cartilage of the limb and trunk skeleton, which originates from the differentiation of condensed mesenchymal cells into clusters of cartilage cells known as chondrocytes. These cartilage anlagen preform the skeleton and provide a framework for endochondral bone development, a process that involves chondrocyte maturation and matrix mineralization in the GPs. The cells of each skeletal element proceed through a multi-step differentiation process generating both the mature GP cartilage, which controls skeletal growth during early and adolescent development, and the permanent articular cartilage (AC) found at the joint surface of all long bones.

The processes of chondrogenesis and endochondral bone formation are not restricted to the developing skeletal system. In fact, following stress-related injuries, such as fractures of endochondral bone, the developmental programs of chondrogenesis and chondrocyte proliferation, maturation, hypertrophy, and terminal differentiation are reinitiated at the site of injury. Additionally, stress-related cartilage diseases such as osteoarthritis (OA) also have marked effects on the differentiation and maintenance

Nonstandard abbreviations used: AC, articular cartilage; Agc, aggrecan; AP, alkaline phosphatase; BMP, bone morphogenetic protein; Col2a1(IIa), the IIa splice form of type II collagen; FOP, fibrodysplasia ossificans progressiva; GP, growth plate; MSC, mesenchymal stem cell; OA, osteoarthritis; SOX9, SRY-box 9; SZ, superficial zone; Tnc, tenascin C

Conflict of interest: The authors have declared that no conflict of interest exists. Citation for this article: J. Clin. Invest. 118:429-438 (2008). doi:10.1172/JCI34174. of AC during adult life. This is why much attention has been paid to studying the cellular and molecular mechanisms that regulate chondrogenesis and chondrocyte differentiation. At the beginning of this Review, we describe the processes of chondrogenesis and chondrocyte differentiation in the hope of highlighting some of the critical molecular regulators of these processes. With this information as a foundation, we provide a synopsis of how several normal and pathologic stressors, including normal mechanical loading, hypoxia, fracture healing, heterotopic ossification, fibrodysplasia ossificans progressiva (FOP), OA, and a number of key environmental factors such as heavy metals and cigarette smoke, impinge upon the progression of these processes. The list of stressors discussed in this Review is not all inclusive, but it represents the most well understood modalities by which stress can have an impact on the chondrogenic and cartilage maturation programs. Overall, understanding the molecular and signaling basis underlying the influence of stress on cartilage will provide the foundation for the development of therapeutic paradigms for ameliorating the impact of pathologic stress in particular.

\section{Cellular events and molecular markers of chondrogenesis and chondrocyte differentiation during endochondral ossification}

Chondrogenesis is a process that is important for the creation of chondrocytes both during embryogenesis as well as in adult life (e.g., during skeletal tissue repair). The process begins with the aggregation and condensation of loose mesenchyme. Factors such as the bone morphogenetic proteins (BMPs) are known to play critical roles in the compaction of mesenchymal cells and the shaping of the condensations (1). During this early step in chondrogenesis, the condensing mesenchyme expresses various ECM and cell adhesion molecules, including the IIa splice form of type II collagen [Col2a1(IIa)] $(2,3), \mathrm{N}$-cadherin (Ncad) (4), N-cam (Ncam1) (5), and tenascin C (Tnc) (6), while also broadly expressing an important transcription factor, SRY-box 9 (Sox9) (Figure 1A, i, and Figure 1B). The Sox family of transcription factors has various roles during chondrogenesis and chondrocyte differentiation, although Sox9 is the primary determinant during the early stages of chondrogenesis $(7,8)$. As the mesenchyme differentiates into chondrocytes, the cells begin to produce an ECM rich in the IIb splice form of type II collagen [Col2a1(IIb)] and aggrecan $(\mathrm{Agc})$. During development, following early chondrocyte differen- 


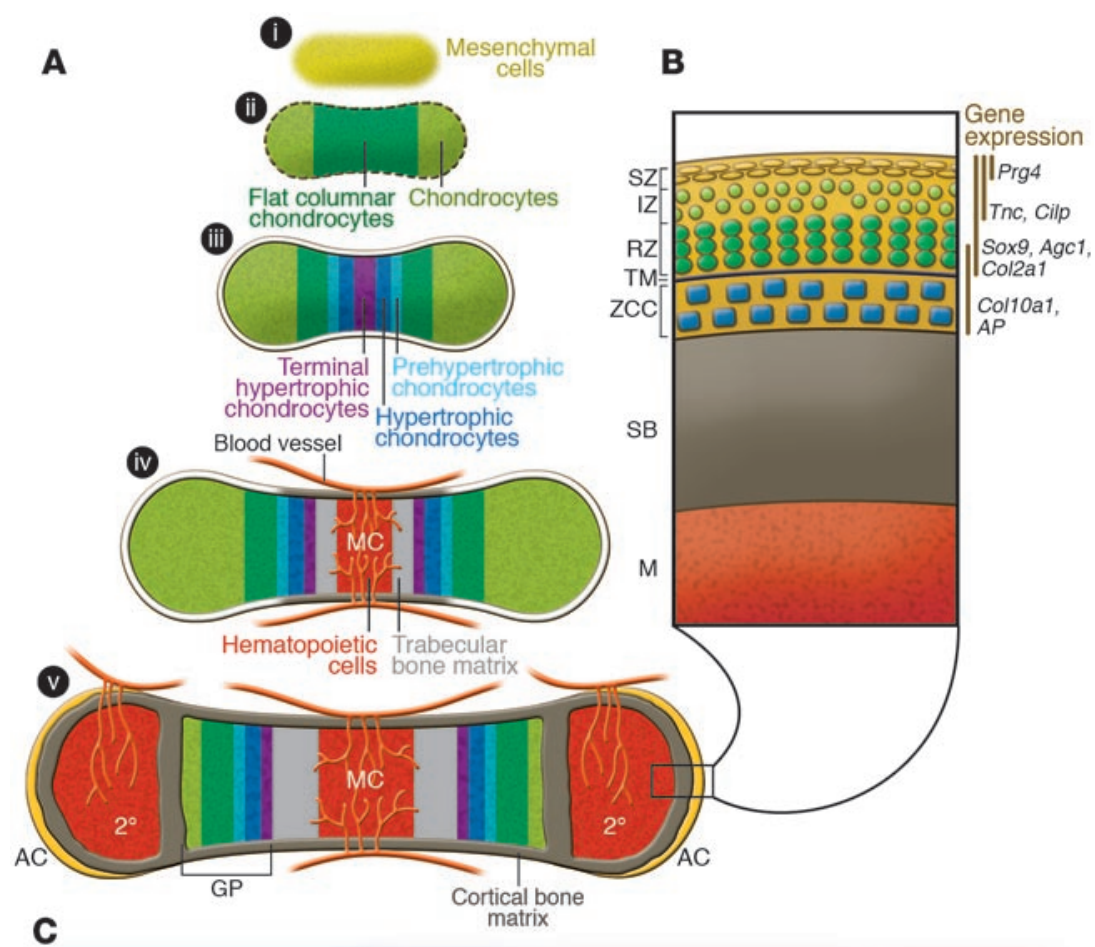

C

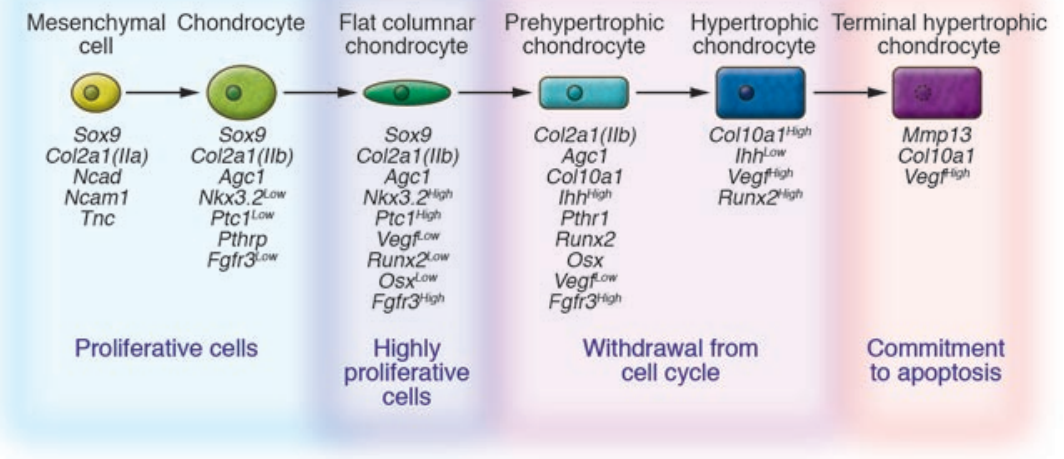

\section{Figure 1}

Cellular events and molecular markers of chondrogenesis, chondrocyte differentiation, and AC development and maintenance. (A) Model of endochondral bone development beginning with mesenchymal cell condensation (i); chondrocyte differentiation and development of the cartilage template (ii); chondrocyte maturation and hypertrophy (iii); separation of cartilage growth regions, vascular invasion, and initiation of both cortical and trabecular bone (iv); and finally generation of the secondary center of ossification that separates AC and GP cartilage during postnatal bone development (v). MC, marrow cavity; $2^{\circ}$, secondary center of ossification. Red lines mark the vasculature, and yellow coloration marks mineralized bone. Black box outlines AC region magnified in B. (B) Graphical representation of the distinct cellular zones in postnatal AC. IZ, intermediate zone; RZ, radial zone; TM, tide mark; ZCC, zone of calcified cartilage; SB, subchondral bone; M, marrow. Vertical lines indicate zones of gene expression. (C) Model outlining the process of chondrogenesis and chondrocyte differentiation. Important markers at each stage of chondrocyte differentiation are listed below the stage at which the genes are expressed. Superscripts indicate the level of gene expression. tiation, the cells rapidly proliferate, enlarging the cartilage templates that preform individual skeletal elements (Figure 1A, i and ii, and Figure 1C). Cells near the center of each growing element eventually withdraw from the cell cycle, initiating the process of hypertrophic differentiation (Figure 1A, iii). During the process of hypertrophic differentiation (i.e., maturation), chondrocytes enlarge, terminally differentiate, mineralize, and ultimately undergo apoptosis. As the chondrocytes die and degrade, their residual cartilage matrix serves as a scaffold for further mineral deposition and bone formation and turnover by invading osteoblasts and osteoclasts. In parallel, the degraded cartilage becomes vascularized by surrounding blood vessels to establish the bone marrow cavity (Figure 1A, iv).

The cartilage GPs located at each end of a developing long bone are generated through a continual process of chondrocyte proliferation, differentiation, and removal. During this process, distinct zones of cells are both morphologically and molecularly identifiable. In the embryo, cells near the distal ends of cartilage elements, periarticular chondrocytes, appear round in shape and express early chondrocyte lineage markers such as Sox9, Col2a1(IIb), Agc1, and low levels of FGF receptor 3 (Fgfr3) (9), as well as specific downstream targets of the indian hedgehog (Ihh) signaling pathway (10-13) (Figure 1A, ii, and Figure 1C). As these cells proliferate and undergo the early steps of maturation, they flatten and form columns parallel to the axis of longitudinal growth. The flat columnar chondrocytes, known to be the most proliferative cells in the cartilage element, express low levels of Runx 2 and Osterix (Osx) and high levels of Fgfr3, Nkx3.2, and Ptc1. Eventually, these columnar cells begin the process of hypertrophy and withdraw from the cell cycle. The prehypertrophic chondrocytes enlarge slightly and initiate expression of $I h h$, parathyroid hormone-related protein receptor $(P T H r P-R)$, as well as increase expression of alkaline phosphatase $(A P)$, and the important regulatory transcription factors Runx 2 and $O s x$, which aid in chondrocyte differentiation as well as being required for mineralization of the cartilage (14-16). As hypertrophy proceeds, the cells continue to enlarge, generate a mineralized matrix, and further enhance their expression of type $\mathrm{X}$ collagen (Col10a1), Runx2, and several growth factors that coordinate chondrocyte proliferation and differentiation. These factors are critical for signaling to the surrounding perichondrial cells to 
induce these cells to differentiate into osteoblast lineage cells that further mineralize the matrix after endochondral ossification is complete. Both hypertrophic chondrocytes and the more terminally differentiated hypertrophic chondrocytes located in the center of the cartilage produce high levels of Vegfa, which is thought to aid in vascularization of the dying cartilage (17). Only the most terminally differentiated hypertrophic chondrocytes express the matrix degrading enzyme Mmp13 (18). MMP13 is an enzyme that controls degradation of the cartilage matrix, a process that precedes mineralization by osteoblasts, that is required for creation of the bone marrow space, and that supports vascular invasion, which provides the cells that will populate the bone marrow (Figure 1A, iii and iv, and Figure 1C). As a point of reference, Figure 1C depicts a summary of the genetic program in chondrocytes undergoing hypertrophic differentiation.

During early postnatal development, epiphyseal chondrocytes (immature chondrocytes located in the center of the epiphyses of long bones) undergo maturation similar to the chondrocyte differentiation process described above that occurs during embryonic skeletogenesis. These cells differentiate, hypertrophy, undergo apoptosis, and are replaced by invading vasculature and osteoblasts, creating the secondary center of ossification (labeled $2^{\circ}$ in Figure 1 ). The secondary center of ossification separates the only two areas of remaining cartilage within individual long bones of the adult skeleton: the $\mathrm{AC}$ and the mature GP cartilage (Figure 1A, v). When chondrogenesis and chondrocyte maturation occur in the adult, such as during fracture repair, no secondary centers of ossification are formed, nor is a novel limb generated, but the processes progress essentially the same way and hypertrophic chondrocytes are ultimately required for analogous purposes - the initiation of mineralization and the induction of vascular invasion.

In the mouse, the development of AC begins during embryogenesis at sites of synovial joint formation. These joints develop through processes including patterning of the joint site, interzone formation, cavitation, and morphogenesis (reviewed in refs. $19,20)$. It has been demonstrated that articular chondrocytes are formed from interzone cells during development. Following embryonic joint formation and postnatal growth, the adult skeleton maintains the cellularity and phenotype of AC via mechanisms largely unknown, whereas GP cartilage completely erodes following adolescent growth in humans. Adult AC is maintained as four distinct cellular zones: the superficial zone (SZ), the intermediate zone (IZ), the radial zone (RZ), and the zone of calcified cartilage (Figure 1B). The SZ consists of 1-2 layers of flattened chondrocytes expressing proteoglycan 4 ( $\operatorname{Prg} 4)$ (also known as SZ protein and lubricin), Sox9, Col2a1(IIb), Agc1, Tnc, and low levels of cartilage intermediate layer protein (Cilp). Chondrocytes of the $\mathrm{IZ}$ are round in appearance and express many of the same molecules as the SZ chondrocytes, although they do not express Prg4 and express higher levels of Cilp. Below the IZ reside the RZ chondrocytes and the zone of calcified cartilage. The RZ chondrocytes express markers of chondrocyte differentiation and hypertrophy such as Col10a1 and AP. Each of the AC regions is normally maintained throughout adulthood unless stress related injury, inflammation, or a genetic defect leads to the loss of either the signals required to maintain these cells or the signals required to inhibit excessive differentiation of these cells. Disruption or impairment of the signals that inhibit excessive differentiation is believed to provide the basis for diseases such as OA.

\section{Effects of stress on chondrogenesis and endochondral ossification during normal skeletal development and maintenance}

Within the framework of the processes of chondrogenesis and chondrocyte differentiation described above and depicted in Figure 1, information about the regulatory impact of normal mechanical and physiologic stress has been published. Presented below is a summary of the influence of normal mechanical and hypoxic stress on the programs of chondrocyte commitment and differentiation.

Mechanical stress. Cellular perception of mechanical stress within cartilaginous tissues is an important modulator of chondrocyte function. This is particularly the case in $\mathrm{AC}$, where the sensing of mechanical forces by chondrocytes leads to profound changes in the health and normal function of the joint $(21,22)$. Mechanical stresses sensed by chondrocytes are often referred to as mechanoelectrochemical events (23), and these coordinate with other environmental, hormonal, and genetic factors to regulate chondrocyte metabolic activity and contribution to the maintenance of the ECM (24). The mechano-electrochemical events sensed by chondrocytes include compressive loading, hydrodynamic/osmotic pressure, fluid flow, ion flow, and electrical current. The generation of these stresses occurs as a coordinated response to mechanical loading and their biophysical nature has been previously reviewed $(23,25)$.

The net impact of mechano-electrochemical stresses on cartilage dynamics is an area of intensive research. Excellent reviews have been published that provide detailed analysis of the anabolism and catabolism that occurs under various mechanical stress situations (see refs. 26, 27). A prevailing hypothesis predicts that the chondrocyte response to moderate mechanical loading is necessary for normal cartilage homeostasis (27). Although static (i.e., constant) loading of cartilage explants in culture leads to the suppression of chondrocyte metabolism and a reduction in chondrocyte Agc and collagen biosynthesis (28), dynamic (i.e., oscillatory) loading at specific frequencies has a net anabolic effect $(26,27)$. Correlating with this, in vivo experiments have shown that changes in joint loading without altering the stability of the tissues (i.e., without injury) can elicit cellular responses that are either catabolic or anabolic depending on frequency, duration, and magnitude of loading (29). Specifically, moderate exercise in young rodents produces an anabolic response in chondrocytes such that cartilage shows increased proteoglycan content, decreased proteoglycan degradation, and increased thickness $(27,29)$. Conversely, high-intensity exercise or a sudden increase in joint loading leads to OA-like catabolism of the cartilage matrix, characterized by decreased collagen networking, proteoglycan loss, and reduced cartilage stiffness (29) (see $O A$ below). Severe inactivity can produce analogous catabolic effects, including reduced cartilage thickness and proteoglycan loss (27). In addition to these effects of mechanical stress on cartilage homeostasis, normal joint loading might also be an important regulator of developmental and postnatal growth of cartilage. Recently published data indicate that abrogation of normal shoulder range of motion via injection of botulinum toxin A into the supraspinatus muscles of newborn mice leads to delayed development of tendonbone insertions (30). Although these results pertain to tendon insertion and/or attachment, it seems plausible to imagine that a similar dysregulation of AC development and/or growth can occur in joints that experience abnormal mechanical loading.

In the context of chondrocyte sensing of mechano-electrochemical events (discussed above), substantial effort has been spent trying to elucidate the key signaling mechanisms facilitating the cel- 


\section{A}

i Periosteum in adults

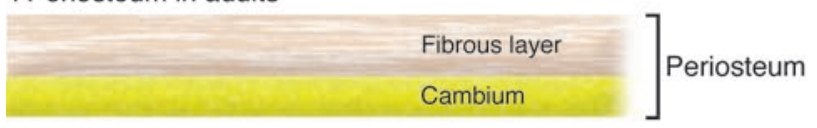

ii Periosteum in children

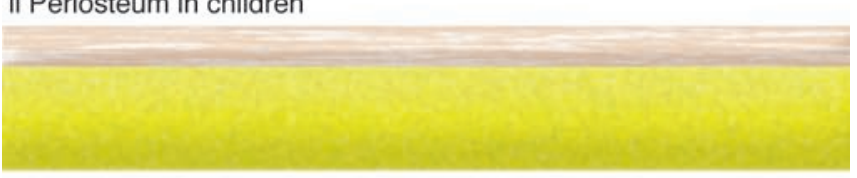

B

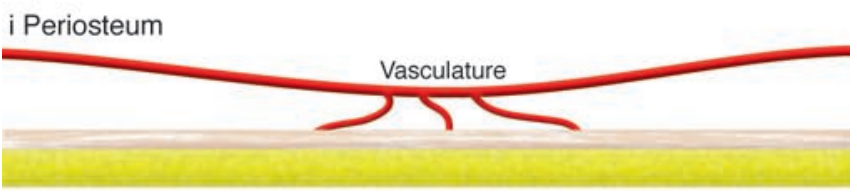

ii Periosteum following injury

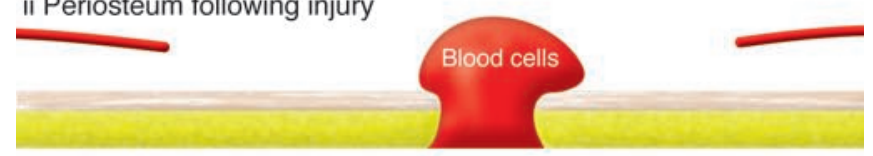

iii Chondrogenesis

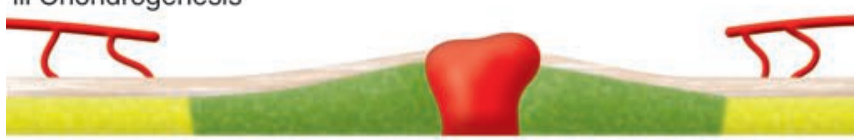

iv Chondrocyte differentiation and intramembranous bone formation

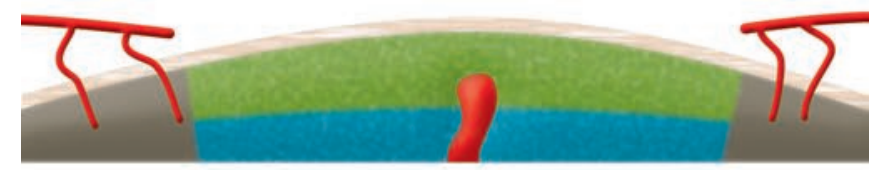

v Chondrocyte maturation and vascular invasion

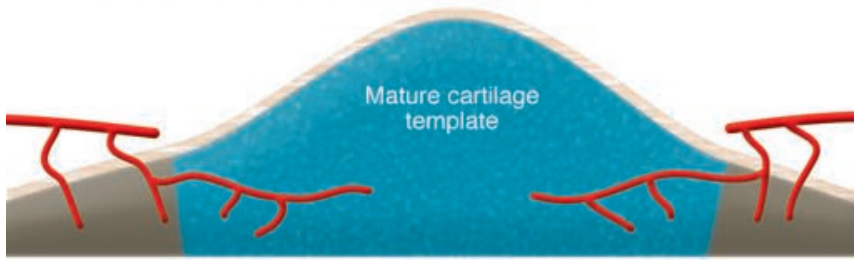

vi Endochondral bone formation

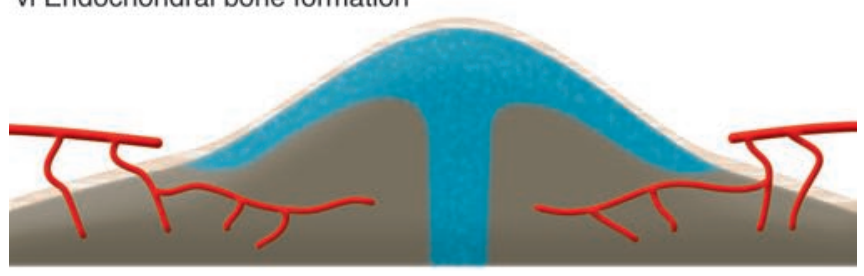

\section{Figure 2}

Contribution of the periosteum to the early phase of fracture healing. (A) Periosteum is a well-microvascularized tissue, consisting of an outer fibrous layer and an inner cambium layer. Children have thicker and better-vascularized cambium layers than adults (i versus ii). (B) The cambium layer contains abundant stem/progenitor cells that can differentiate into bone and cartilage (i). Following fracture or osteotomy, progenitor cells residing in the periosteum are activated and enter the cell cycle (ii), followed by differentiation into osteoblastic and chondrogenic (green) lineage cells (iii). Further differentiation of the osteoblasts and chondrocytes leads to intramembranous bone formation (gray) and a mature cartilage template (blue) (iv). The vascular invasion of the cartilage template (v) coupled with bone formation completes the process of endochondral bone repair (vi).

lular effects of loading. A central signal transduction pathway that regulates these processes involves the integrin receptors. Integrin receptor complexes bind ECM components and transmit information about mechanical perturbations of the ECM to the cytoplasm of chondrocytes. Integrin receptors containing a $\beta 1$ subunit are the primary integrins responsible for binding the ECM (31). For example, an $\alpha_{1} \beta_{1}$ or $\alpha_{2} \beta_{1}$ integrin complex typically binds collagen and laminin, the $\alpha_{3} \beta_{1}$ integrin complex binds fibronectin and type II collagen, and the $\alpha_{V} \beta_{1}$ integrin complex is the classical receptor for fibronectin (reviewed in ref. 25). Adhesion and binding of ECM components to integrin receptors on the surface of chondrocytes leads to the activation of proteins associated with the cytoskeleton, such as paxillin (32), and intracellular signaling proteins, such as focal adhesion kinase (FAK) (33) and MAPK signaling molecules (34). In fact, MAPK signaling with downstream activation of the MEK-Erk1 signaling pathway leads to downregulation of Agc gene expression in bovine articular chondrocytes (35). Furthermore, association of the integrin complex with IGF receptor I strongly facilitates activation of this MAPK signaling pathway (34). There is also evidence for activation of proline-rich tyrosine kinase 2 (Pyk2), leading to the upregulation of collagenase III expression via PKC following exposure to fibronectin fragments (36). In addition to these signaling responses, it is important to note that abrogation of cell-ECM interactions (anoikis) that are mediated via integrins leads to chondrocyte apoptosis (37), establishing a true dependence of chondrocyte function on sensing mechanical forces transmitted by the ECM.

It is widely thought that transduction of mechano-electrochemical events can also be facilitated via stress-activated ion channels in the plasma membranes of chondrocytes. Of the numerous ion channels that have been characterized, the most relevant in chondrocytes may be the $\mathrm{N}$ - and L-type voltage-gated calcium channels (VGCCs) $(38,39)$. Since cytoskeletal elements control opening and closing of VGCCs in neuronal cells, a similar regulatory paradigm might exist in chondrocytes. Thus, transfer of mechanical stress through the cytoskeleton could induce opening of these channels, the propagation of intracellular calcium waves, and the subsequent induction of phenotypic effects in the cells (40). In general, calcium transients lead to activation of signaling via both calmodulin kinase and calcineurin/NFAT pathways $(41,42)$ among others. Although these pathways are known to be important in the modulation of both chondrogenesis and chondrocyte differentiation $(43,44)$, work remains to fully characterize how calcium signaling in chondrocytes contributes to the anabolic or catabolic effects of mechanical stress. 
Hypoxic stress. Oxygen is required for normal cellular metabolism, and oxygen deficiency within cartilage tissues can induce an hypoxic state that affects chondrocyte function. Cells exposed to hypoxia respond in a number of ways, including altering their expression of genes involved in cell cycle, differentiation, and apoptosis (45). In mesenchymal stem cells (MSCs) and chondrocytes, the transcription factor HIF-1 $\alpha$ is a survival factor that is induced in hypoxic environments and inhibits proliferation but increases ECM production $(46,47)$. Conditional deletion of the gene encoding HIF- $1 \alpha$ in chondrocytes results in massive apoptosis in hypoxic areas (46). Three-dimensional micromass cultures of adipose-derived adult stromal cells with targeted deletion of the gene encoding HIF-1 $\alpha$ have substantially reduced chondrogenic potential (48), suggesting that hypoxia-induced upregulation of this factor supports chondrogenic commitment. Thus, hypoxia is likely to be an important stressor that enhances the chondrogenic potential of mesenchymal cell populations both during the development of normal tissues that have limited blood supply as well as under pathologic conditions (e.g., following disruption of vascularity due to injury).

\section{The influence of pathologic stress on chondrogenesis and endochondral ossification}

More is known about how cartilage biology is influenced by stressors that arise from pathologic situations than is known about how cartilage biology is affected by normal physiologic stressors. Below is a compilation of key information regarding the influence on chondrogenesis and chondrocyte differentiation of pathologic stress induced by fracture healing, OA, heterotopic ossification, FOP, heavy metal toxicity, and cigarette smoke.

Fracture healing. Skeletal fracture is an important pathologic stress that has a critical impact on the chondrogenesis and endochondral ossification that occurs during the fracture healing response. The mechanisms underlying the initiation of chondrocyte progenitor proliferation and differentiation at the time of fracture injury are not well understood, although it has been suggested that mechanical stability and the early inflammatory response play key roles (49). Following cortical bone fracture or osteotomy, local progenitor cells residing in either the periosteum or bone marrow are sensitized, enabling them to respond to biological or biophysical stimuli produced within the local injury milieu. During the first few days following fracture, a hematoma is formed and this is followed by infiltration of inflammatory cells and release of growth factors, which direct the recruitment and proliferation of progenitor cells (Figure 2). The further differentiation of the recruited progenitor cells into chondrocyte and/or osteoblast lineage cells determines the fate of the cells - whether they enter the endochondral bone formation pathway or the intramembranous bone formation pathway. It is evident that the environment at the site of the injury, for example the presence of hypoxia and inflammation, as well as the mechanical stability of the bone ends affects the fate decision of the progenitor cells. In particular, endochondral bone formation, which is the focus of this Review, always takes place close to where the junction between the broken bone ends will develop. This is where the oxygen tension is low and vascularity is severely damaged. In terms of mechanical stability, stabilized fractures heal with virtually no evidence of cartilage (intramembranous bone formation), whereas nonstabilized fractures produce abundant cartilage at the fracture site (endochondral bone formation). As mentioned earlier, the schematic in Figure 2 shows the unique morphogenesis of reparative tissue during the early phases of bone fracture healing via endochondral bone formation, and this has been detailed in excellent review articles (see refs. 50, 51).

The progenitor cells that contribute to the fracture healing response are found in both the bone marrow and, to a greater extent, in the periosteum. Periosteum consists of microvascularized connective tissue that covers the outer surface of cortical bone. It can be separated into two distinct layers: an outer layer that contains fibroblasts and distinct connections between the periosteum and bone, which are known as Sharpey fibers; and an inner layer known as cambium, which contains multipotent MSCs and osteoprogenitor cells that contribute to normal bone growth, healing, and regeneration (52-54). It is known that the cambium layer in children is much thicker and better vascularized than in adults, a possible underlying cause for faster and more complete healing of fractures in children.

Due to the lack of cellular markers of pluripotent MSCs, the identity of the multipotent stem/progenitor cells residing in the periosteum are largely unknown. This substantially hampers efforts to track the fate of these cells in repair and further understand the cellular and molecular mechanism(s) pertaining to the activation of this important progenitor cell pool. A murine segmental bone graft transplantation model that includes periosteum $(55,56)$ has permitted examination of the signals and stem cell populations involved in bone repair in transgenic and knockout mice. When live bone grafts derived from mice constitutively expressing $\beta$-galactosidase in all tissues were transplanted to wild-type mice, it was observed that about $70 \%$ of the early bone and cartilage formation overlying the bone graft was attributable to the proliferation and differentiation of donor periosteal progenitors (55). These data strongly suggest that live cortical bone healing is initiated and driven by pluripotent local MSCs. Using the live bone transplantation approach in transgenic and knockout mice should provide an in vivo model to examine the influence of injury stress on progenitor cell activation, proliferation, and differentiation.

The molecular signaling pathways involved in the initiation and morphogenesis of fracture repair are only superficially understood. Experimental data suggest that periosteum-initiated bone repair might be analogous to fetal limb bud development $(57,58)$. The mesenchymal condensation that forms at the fracture site that is analogous to the developing limb bud (blastema) originates from the extensive proliferation of progenitor cells at the injury site (59). Although animals and humans have only very limited capacity to regenerate damaged tissues, it has long been suspected that postnatal bone repair, such as fracture healing, recapitulates some of the essential pathways in limb development, although the fracture repair process is not capable of supporting limb regeneration per se.

In the past decade, progress has been made in the identification of factors and genes involved in fracture healing. The most notable of these are BMPs, which belong to the TGF- $\beta$ superfamily, hedgehog proteins, and Wnt proteins. For example, BMP-2 expression was found in the early fracture callus (i.e., the tissue that forms at the fracture site; see Figure 2) just a few days following cortical bone fracture (60). Most recently, Tsuji et al. demonstrated that elimination of BMP-2 in the mouse limb disrupted the initiation of postnatal fracture healing (61), indicating an essential role for BMP-2 in bone repair and healing. Evidence has also emerged to show that morphogens such as hedgehog proteins and Wnt proteins, which are involved in embryonic pattern formation, also 
postembryonically function to initiate pathways that control selfrenewal, migration, differentiation, and cell-fate commitment of adult stem or progenitor cells (62). However, detailed studies are needed to determine the role of these two pathways in fracture healing in adults.

In addition to mechanisms analogous to limb development, genes that are involved in injury and inflammatory responses during the repair process have been shown to play key roles in endochondral bone repair $(63,64)$. Nonsteroidal antiinflammatory drugs (NSAIDs) that target COX isoforms are the most commonly used pain-relieving medications in our society. Several studies have shown that COX activity is involved in normal bone metabolism and suggested that NSAIDs have a negative impact on bone repair $(65,66)$. The most compelling data implicating COX activity in bone repair come from genetic models that demonstrate a critical role for the inducible COX isoform COX-2 - although they develop normally, bone repair is markedly impaired in adult Cox $-2^{-/-}$mice following fracture (67). Defects occur at various stages of healing, including during chondrogenesis and chondrocyte differentiation. Histology of the fracture callus, derived from Cox $-2^{-/-}$mice given a tibia fracture, shows delayed chondrogenesis and persistent mesenchymal cells at the junction of the fracture site. The point during the bone healing process that is impaired in Cox-2-/- mice coincides with the early induction of COX-2 expression following bone fracture in wild-type mice, further demonstrating the requirement of this enzyme in early chondrogenesis during skeletal repair.

Heterotopic ossification. A well-characterized pathologic stress response of muscle to injury involves the development of heterotopic ossification. Ectopic bone formation develops through a cartilage intermediate, so the initial events of heterotopic ossification require chondrogenesis. Muscle injury results in tissue damage, hemorrhage, and an inflammatory response. Recent data have suggested that the development of a hypoxic environment is critical if the early activation tissue response is to lead to chondrogenesis (68). Although the subsequent induction of BMPs in the local environment has been connected with the formation of ectopic bone in both animal models (69-72) and humans (73), the manner in which stress signals regulate BMP expression and enhance the BMP signaling response is not clear. However, the finding that noggin, gremlin, and follistatin (inhibitors of BMP signaling) can suppress the development of ectopic bone in animal models suggests that control of BMP signaling in injured tissues is a critical event $(74,75)$.

Signals downstream of the stress response have been implicated in regulating the BMP signaling pathway. For example, inhibition of COX-2-dependent stimulation of $\mathrm{PGE}_{2}$ production from human bone marrow-derived MSCs using NS-398 suppresses expression of BMP-2 (76). This suppression can be reversed by an agonist of the EP4 receptor for $\mathrm{PGE}_{2}$ as well as by $\mathrm{PGE}_{2}$. In addition, BMP-2 expression is also suppressed by an EP4 receptor antagonist in these cells (76). Based on this, COX-2/PGE $/$ EP4 receptor/BMP-2 signaling in MSCs is likely to be important in heterotopic bone formation. Recent reports also demonstrate that BMP-2 stimulates Osx mRNA expression and AP activity in ST2 pluripotent stromal cells, and these effects are enhanced by the selective EP4 receptor agonist ONO-4819 (77). In this study, pretreatment of the ST2 cells with a PKA inhibitor abolished the effects of ONO-4819, suggesting that the anabolic effect of activation of the EP4 receptor is mediated by the PKA pathway. Thus,
COX-2 induction of $\mathrm{PGE}_{2}$ production and signaling through PKA in muscle stem cells is a possible link between the inflammatory response to injury and ectopic bone formation. Although these studies have not involved the use of muscle stem cells, recent human data demonstrating that inhibition of COX-2 prevents heterotopic bone formation following hip arthroplasty are consistent with these findings (78-80).

FOP. FOP is a rare and disabling genetic disorder characterized by the inappropriate deposition of cartilage leading to progressive heterotopic ossification. Throughout childhood and early adult life, the joints of the normotopic skeleton of individuals with FOP become progressively immobilized, rendering movement impossible. Any small injury to connective tissue (muscles, ligaments, and tendons) typically results in the formation of hard bone around the damaged area. FOP is an autosomal dominant condition, but most cases are sporadic. Recently, a recurrent mutation in the glycine-serine activation domain of the type IA activin receptor gene (ACVR1) was reported in all sporadic and familial cases of classic FOP, indicating that this activating mutation of the ACVR1 gene is responsible for the disease (81). It has been additionally shown that BMP-4 and type IA BMP receptor proteins are overexpressed in cultured lymphocytes from FOP patients (82). Recent studies have shown that stem cells isolated from the skeletal muscle of mice exhibit long-term proliferation, high self-renewal, multipotent differentiation, and have osteogenic potential (83-85). Overexpression of BMP-4 induces these muscle-derived stem cells to acquire a chondrogenic phenotype in vitro (84). One conceivable explanation for FOP is that MSCs in the area surrounding a future site of heterotopic bone are not prone to differentiate into bone-forming osteogenic cells under normal conditions but that mutations in ACVR1 in these tissues trigger mesenchymal cells to differentiate into cells that support heterotopic endochondral ossification.

$O A$. OA, the most common form of arthritis, is a noninflammatory degenerative joint disease characterized by dysfunction of articular chondrocytes, AC degradation, periarticular bone formation (i.e., osteophytes), and enhanced bone density below the AC surface (i.e., subchondral sclerosis) (86). Although the etiology of $\mathrm{OA}$ is not fully understood, it is generally held that biochemical, genetic, and mechanical factors participate in the progression of the $\mathrm{AC}$ degeneration (87). In the early stages of disease, the production of catabolic cytokines by the membrane surrounding the joint capsule (i.e., the synovium) induces transient AC proliferation and increased matrix synthesis (type II collagen, Agc) in an attempt to initiate repair $(88,89)$. However, the chronic production of these cytokines, which include IL-1, TNF- $\alpha$, IL-17, and IL-18 as well as $\mathrm{PGE}_{2}$, leads to the enhanced synthesis of collagenases (MMP-1, MMP-8, MMP-9, and MMP-13) and aggrecanases (ADAMTS4 and ADAMTS5), which drive matrix degradation and progressively erode the articular surface $(87,90,91)$. Progressive degradation of the matrix is associated with the dysregulation of AC, denoted by the inappropriate expression of genetic and morphologic markers of endochondral ossification, including type X collagen, AP, and increased apoptosis $(90,92)$. It has been hypothesized that this alteration of AC phenotype, leading to disruption of normal matrix anabolism, is an initiating step in the disease process. Of particular interest, from the perspective of this Review, is the influence of cytokines and abnormal mechanical stress on the degeneration of the $\mathrm{AC}$ at the onset and during the progression of OA.

As mentioned in the above discussion of mechanical loading of cartilage, high-intensity exercise, abnormally large static loading, 
and a sudden increase in joint loading all lead to OA-like matrix catabolism characterized by decreased collagen networking, proteoglycan loss, and reduced cartilage stiffness (29). Alterations in joint mechanics that are caused by injury to cartilage or surrounding structures (such as the shock-absorbing meniscus in the knee) would presumably also lead to this type of cartilage catabolism. This would be due to both the production of cytokines as well as the abnormal mechanical loading of the joint caused by tissues damaged by injury (reviewed in ref. 27). Specifically, cytokines such as IL- 1 and TNF- $\alpha$ are inducers of cartilage matrix degradation due to their ability to induce the expression of genes encoding matrix catabolizing proteins such as MMPs and aggrecanases (93, 94). In fact, both IL-1 and TNF- $\alpha$ induce $\mathrm{PGE}_{2}$ production and $\mathrm{NO}$ metabolism, which both act as strong catabolic signals in cartilage by promoting chondrocyte injury and enhancing chondrocyte apoptotic potential (95).

Articular chondrocytes might also respond directly to cytokines and abnormal mechanical loading via the generation of intracellular ROS, which also is likely to contribute to the pathogenesis of OA $(96,97)$. Generation of ROS is associated with enhanced production of MMPs and cytokines (98) and with concomitant enhancement of NF-KB signaling (99). When in excess, ROS (e.g., superoxide anion and hydrogen peroxide) induce apoptosis by oxidative stress and/or damage to DNA, lipids, and proteins. In the context of endochondral ossification (and possibly in chondrocytes that are undergoing inappropriate hypertrophy during OA), ROS are increased as the cells differentiate (100). Correlated with this, treatment of chondrocytes with oxidants induces hypertrophy, whereas antioxidant treatment with $N$-acetyl cysteine inhibits maturation with decreased expression of the maturation markers Mmp13, Col10a1, and Runx2 (100). Maturing hypertrophic chondrocytes release matrix vesicles, and this event is increased following hydrogen peroxide treatment, supporting the hypothesis that ROS induce hypertrophy (101). Given these findings, the use of antioxidant therapy as a possible therapeutic paradigm in $\mathrm{OA}$ has been discussed (102).

Heavy metals. Lead $(\mathrm{Pb})$, the heavy metal most widely studied in biological systems, has toxic effects on various organs including the skeleton (103-105). Bone is a major reservoir for ingested $\mathrm{Pb}$ (106), and delayed skeletal development occurs in children with prenatal exposure to $\mathrm{Pb}(107)$. In addition, levels of exposure to $\mathrm{Pb}$ during childhood are inversely correlated with final height, weight, and chest circumference (108-110). These observations suggest a direct effect of $\mathrm{Pb}$ on skeletal development, with the possibility that there are direct effects of this heavy metal on chondrogenesis and endochondral ossification.

$\mathrm{Pb}$ stimulates chondrogenesis in micromass cultures of murine mesenchymal limb buds and during in vivo ectopic bone formation and fracture healing. Regarding the in vitro work, $\mathrm{Pb}$-exposed micromass cultures of primary limb bud MSCs have increased Sox9 and Col2 expression as well as cartilage nodule formation (111). $\mathrm{Pb}$ causes pathway-specific effects on these cells, including enhanced phosphorylation of the TGF- $\beta$-specific Smads (Smad 2 and Smad3) and decreased phosphorylation of the BMP-2-specific Smads (Smad1, Smad5, and Smad8) (111). The increase in cartilage formation seems, in part, to be secondary to increased mesenchymal cell proliferation. Correlating with this, mice exposed to $\mathrm{Pb}$ with blood $\mathrm{Pb}$ levels mimicking the established childhood toxicity range have accelerated and increased chondrogenesis in muscles implanted with BMP-2-expressing C9 cells (111). However, during the endochondral ossification phase of ectopic bone formation in this model, $\mathrm{Pb}$ inhibits chondrocyte maturation (111). Since BMP signaling is essential for chondrocyte maturation, it is possible that the effect is secondary to downregulation of this signaling pathway, as was observed in vitro. Thus, although $\mathrm{Pb}$ stimulates chondrogenesis, it reduces chondrocyte maturation and thereby results in delayed endochondral bone formation, as has been previously documented (112). Consistent with these in vitro and in vivo data, $\mathrm{Pb}$ exposure has been found to inhibit fracture healing in mice, with complex effects noted on chondrogenesis and chondrocyte maturation (113). The enhanced cartilage deposition and delayed mineralization (i.e., delayed completion of endochondral ossification) seen in this fracture experiment further support the impact of stress related to $\mathrm{Pb}$ toxicity on chondrogenesis and chondrocyte maturation.

Only a few reports address the chondrogenic effect of exposure to other heavy metals. Cadmium induces apoptosis of mesenchymal populations adjacent to the apical ectodermal ridge (114), reducing the cartilage-forming potential of the tissue in the developing limb. Similarly, quantum dots comprised of a combination of cadmium, selenium, and zinc inhibit chondrogenesis in bone marrow progenitors (115). Another report documents the influence of zinc, manganese, and cadmium on chondrocytes during endochondral ossification (98). Zinc was found to have a biphasic effect on chondrocyte differentiation, with low doses inducing AP activity and high doses inhibiting mineralization (116). Comparatively, manganese caused a reduction of mineralization, whereas the effects of cadmium were acutely toxic (116). Although these heavy metals clearly regulate chondrogenesis, little is known about the molecular mechanisms underlying the toxicity in mesenchymal populations that leads to impaired cartilage formation.

Cigarette smoke. In addition to effects on lung, heart, and vascular tissue, smoking has been documented to have a negative impact on skeletal healing (117-124). Cartilage is an important target of cigarette smoke during fracture repair, and effects are observed on mesenchymal cell recruitment to the chondrocyte lineage and the later stages of endochondral bone formation. For example, exposure of mice to second-hand smoke caused delayed chondrogenesis in a tibial fracture model, as evidenced by reduced toluidine blue staining at day 7 after fracture and peak cartilage formation at day 14 when control calluses are mineralized (125). Work to identify which components of cigarette smoke are responsible for this inhibitory effect on chondrogenesis is important if the underlying molecular mechanisms are to be elucidated.

\section{Conclusions}

Overall, normal and pathologic stress substantially affect the differentiation of chondroprogenitors and chondrocytes, and thus exert critical influence over cartilage tissue homeostasis. As can be seen from this Review, the bulk of our knowledge derives from work that has identified the influence of various pathologic stresses, including skeletal fracture, OA, heterotopic ossification, and heavy metal toxicity. Ongoing efforts to further address how pathologic stress affects cartilage are evident in the literature and driven by the strong interest in understanding the control of cartilage biology in the context of trauma, inflammation, and healing. Regarding the influence of normal stresses on cartilage (in particular, mechanical stress), clear paradigms have been established that support a signal transduction scheme for stress to exert cell effects. How these stresses affect the formation of limbs, AC, and 
joints is not well understood, and represents an important area of future research. Understanding how the subtle application of normal mechanical stress leads to appropriate limb and cartilage development is important for envisioning therapies that facilitate cartilage repair, including tissue engineering approaches.
Address correspondence to: Regis J. O’Keefe, Box 665, Department of Orthopaedics, University of Rochester Medical Center, 601 Elmwood Avenue, Rochester, New York 14642, USA. Phone: (585) 273-1261; Fax: (585) 275-1121; E-mail: regis_okeefe@ urmc.rochester.edu.
1. Barna, M., and Niswander, L. 2007. Visualization of cartilage formation: insight into cellular properties of skeletal progenitors and chondrodysplasia syndromes. Dev. Cell. 12:931-941.

2. Ng, L.J., Tam, P.P., and Cheah, K.S. 1993. Preferential expression of alternatively spliced mRNAs encoding type II procollagen with a cysteine-rich amino-propeptide in differentiating cartilage and nonchondrogenic tissues during early mouse development. Dev. Biol. 159:403-417.

3. Sandell, L.J., Morris, N., Robbins, J.R., and Goldring, M.B. 1991. Alternatively spliced type II procollagen mRNAs define distinct populations of cells during vertebral development: differential expression of the amino-propeptide. J. Cell Biol. 114:1307-1319.

4. Oberlender, S.A., and Tuan, R.S. 1994. Expression and functional involvement of $\mathrm{N}$-cadherin in embryonic limb chondrogenesis. Development. 120:177-187

5. Widelitz, R.B., Jiang, T.X., Murray, B.A., and Chuong, C.M. 1993. Adhesion molecules in skeletogenesis: II. Neural cell adhesion molecules mediate precartilaginous mesenchymal condensations and enhance chondrogenesis. J. Cell. Physiol. 156:399-411.

6. Mackie, E.J., Thesleff, I., and Chiquet-Ehrismann, R. 1987. Tenascin is associated with chondrogenic and osteogenic differentiation in vivo and promotes chondrogenesis in vitro. J. Cell Biol. 105:2569-2579.

7. Akiyama, H., Chaboissier, M.C., Martin, J.F., Schedl, A., and de Crombrugghe, B. 2002. The transcription factor Sox9 has essential roles in successive steps of the chondrocyte differentiation pathway and is required for expression of Sox 5 and Sox 6 . Genes Dev. 16:2813-2828.

8. Bi, W., et al. 2001. Haploinsufficiency of Sox9 results in defective cartilage primordia and premature skeletal mineralization. Proc. Natl. Acad. Sci. U. S. A. 98:6698-6703.

9. Deng, C., Wynshaw-Boris, A., Zhou, F., Kuo, A., and Leder, P. 1996. Fibroblast growth factor receptor 3 is a negative regulator of bone growth. Cell. 84:911-921.

10. Vortkamp, A., et al. 1996. Regulation of rate of cartilage differentiation by Indian hedgehog and PTH-related protein. Science. 273:613-622.

11. St-Jacques, B., Hammerschmidt, M., and McMahon, A.P. 1999. Indian hedgehog signaling regulates proliferation and differentiation of chondrocytes and is essential for bone formation. Genes Dev 13:2072-2086.

12. Long, F., Zhang, X.M., Karp, S., Yang, Y., and McMahon, A.P. 2001. Genetic manipulation of hedgehog signaling in the endochondral skeleton reveals a direct role in the regulation of chondrocyte proliferation. Development. 128:5099-5108.

13. Hilton, M.J., Tu, X., and Long, F. 2007. Tamoxifeninducible gene deletion reveals a distinct cell type associated with trabecular bone, and direct regulation of PTHrP expression and chondrocyte morphology by Ihh in growth region cartilage. Dev. Biol. 308:93-105.

14. Inada, M., et al. 1999. Maturational disturbance of chondrocytes in Cbfa1-deficient mice. Dev. Dyn 214:279-290.

15. Yoshida, C.A., et al. 2004. Runx2 and Runx3 are essential for chondrocyte maturation, and Runx2 regulates limb growth through induction of Indian hedgehog. Genes Dev. 18:952-963.

16. Nakashima, K., et al. 2002. The novel zinc finger- containing transcription factor osterix is required for osteoblast differentiation and bone formation. Cell. 108:17-29.

17. Gerber, H.P., et al. 1999. VEGF couples hypertrophic cartilage remodeling, ossification and angiogenesis during endochondral bone formation. Nat. Med. 5:623-628.

18. Mattot, V., et al. 1995. Expression of interstitial collagenase is restricted to skeletal tissue during mouse embryogenesis. J. Cell Sci. 108:529-535.

19. Archer, C.W., Dowthwaite, G.P., and Francis-West, P. 2003. Development of synovial joints. Birth Defects Res. C Embryo Today. 69:144-155.

20. Pacifici, M., Koyama, E., and Iwamoto, M. 2005 Mechanisms of synovial joint and articular cartilage formation: recent advances, but many lingering mysteries. Birth Defects Res. C Embryo Today. 75:237-248.

21. Buckwalter, J.A., and Mankin, H.J. 1998. Articular cartilage: tissue design and chondrocyte-matrix interactions. Instr. Course Lect. 47:477-486.

22. Guilak, F. 2000. The deformation behavior and viscoelastic properties of chondrocytes in articular cartilage. Biorbeology. 37:27-44.

23. Mow, V.C., Wang, C.C., and Hung, C.T. 1999. The extracellular matrix, interstitial fluid and ions as a mechanical signal transducer in articular cartilage. Osteoarthr. Cartil. 7:41-58.

24. Mobasheri, A., et al. 1998. Ion transport in chondrocytes: membrane transporters involved in intracellular ion homeostasis and the regulation of cell volume, free $[\mathrm{Ca} 2+]$ and $\mathrm{pH}$. Histol. Histopathol. 13:893-910.

25. Mobasheri, A., Carter, S.D., Martin-Vasallo, P., and Shakibaei, M. 2002. Integrins and stretch activated ion channels; putative components of functional cell surface mechanoreceptors in articular chondrocytes. Cell Biol. Int. 26:1-18.

26. Guilak, F., Sah, R.L., and Setton, L.A. 1997. Physical regulation of cartilage metabolism. In Basic orthopaedic biomechanics. V.C. Mow and W.C. Hayes, editors. Lippincott-Raven. Philadelphia, Pennsylvania, USA. 197-207.

27. Griffin, T.M., and Guilak, F. 2005. The role of mechanical loading in the onset and progression of osteoarthritis. Exerc. Sport Sci. Rev. 33:195-200.

28. Buschmann, M.D., Hunziker, E.B., Kim, Y.J., and Grodzinsky, A.J. 1996. Altered aggrecan synthesis correlates with cell and nucleus structure in statically compressed cartilage. J. Cell Sci. 109:499-508.

29. Arokoski, J.P., Jurvelin, J.S., Vaatainen, U., and Helminen, H.J. 2000. Normal and pathological adaptations of articular cartilage to joint loading. Scand. J. Med. Sci. Sports. 10:186-198.

30. Thomopoulos, S., et al. 2007. Decreased muscle loading delays maturation of the tendon enthesis during postnatal development. J. Orthop. Res. 25:1154-1163.

31. Shakibaei, M., De Souza, P., and Merker, H.J. 1997. Integrin expression and collagen type II implicated in maintenance of chondrocyte shape in monolayer culture: an immunomorphological study. Cell Biol. Int. 21:115-125.

32. Burridge, K., Turner, C.E., and Romer, L.H. 1992. Tyrosine phosphorylation of paxillin and pp125FAK accompanies cell adhesion to extracellular matrix: a role in cytoskeletal assembly. J. Cell Biol. 119:893-903.

33. Schaller, M.D., Otey, C.A., Hildebrand, J.D., and Parsons, J.T. 1995. Focal adhesion kinase and paxillin bind to peptides mimicking beta integrin cyto- plasmic domains. J. Cell Biol. 130:1181-1187.

34. Shakibaei, M., John, T., De Souza, P., Rahmanzadeh, R., and Merker, H.J. 1999. Signal transduction by beta 1 integrin receptors in human chondrocytes in vitro: collaboration with the insulin-like growth factor-I receptor. Biochem. J. 342:615-623.

35. Hung, C.T., et al. 2000. Mitogen-activated protein kinase signaling in bovine articular chondrocytes in response to fluid flow does not require calcium mobilization. J. Biomech. 33:73-80.

36. Loeser, R.F., Forsyth, C.B., Samarel, A.M., and Im, H.J. 2003. Fibronectin fragment activation of proline-rich tyrosine kinase PYK2 mediates integrin signals regulating collagenase- 3 expression by human chondrocytes through a protein kinase C-dependent pathway. J. Biol. Chem. 278:24577-24585.

37. Cao, L., et al. 1999. beta-Integrin-collagen interaction reduces chondrocyte apoptosis. Matrix Biol. 18:343-355.

38. Guilak, F., et al. 1999. Mechanically induced calcium waves in articular chondrocytes are inhibited by gadolinium and amiloride. J. Orthop. Res. 17:421-429.

39. Wang, X.T., et al. 2000. Cardiac L-type calcium channel alpha 1 -subunit is increased by cyclic adenosine monophosphate: messenger RNA and protein expression in intact bone. J. Bone Miner. Res. 15:1275-1285.

40. Chao, P.H., West, A.C., and Hung, C.T. 2006. Chondrocyte intracellular calcium, cytoskeletal organization, and gene expression responses to dynamic osmotic loading. Am. J. Physiol. Cell Physiol. 291:C718-C725.

41. Zayzafoon, M. 2006. Calcium/calmodulin signaling controls osteoblast growth and differentiation. J. Cell. Biochem. 97:56-70.

42. Rao, A., Luo, C., and Hogan, P.G. 1997. Transcription factors of the NFAT family: Regulation and function. Annu. Rev. Immunol. 15:707-747.

43. Huser, C.A., and Davies, M.E. 2007. Calcium signaling leads to mitochondrial depolarization in impactinduced chondrocyte death in equine articular cartilage explants. Arthritis Rheum. 56:2322-2334.

44. Tomita, M., Reinhold, M.I., Molkentin, J.D., and Naski, M.C. 2002. Calcineurin and NFAT4 induce chondrogenesis. J. Biol. Chem. 277:42214-42218.

45. Schipani, E. 2005. Hypoxia and HIF-1 alpha in chondrogenesis. Semin. Cell Dev. Biol. 16:539-546.

46. Schipani, E., et al. 2001. Hypoxia in cartilage: HIF1 alpha is essential for chondrocyte growth arrest and survival. Genes Dev. 15:2865-2876.

47. Pfander, D., Cramer, T., Schipani, E., and Johnson, R.S. 2003. HIF-1alpha controls extracellular matrix synthesis by epiphyseal chondrocytes. J. Cell Sci. 116:1819-1826.

48. Malladi, P., Xu, Y., Chiou, M., Giaccia, A.J., and Longaker, M.T. 2006. Effect of reduced oxygen tension on chondrogenesis and osteogenesis in adipose-derived mesenchymal cells. Am. J. Physiol. Cell Physiol. 290:C1139-C1146.

49. Le, A.X., Miclau, T., Hu, D., and Helms, J.A. 2001. Molecular aspects of healing in stabilized and nonstabilized fractures. J. Orthop. Res. 19:78-84.

50. Einhorn, T.A. 1998. The cell and molecular biology of fracture healing. Clin. Orthop. 355(Suppl.):S7-S21.

51. Gerstenfeld, L.C., Cullinane, D.M., Barnes, G.L., Graves, D.T., and Einhorn, T.A. 2003. Fracture healing as a post-natal developmental process: molecular, spatial, and temporal aspects of its regulation. J. Cell. Biochem. 88:873-884.

52. Augustin, G., Antabak, A., and Davila, S. 2007. The 
periosteum Part 1: Anatomy, histology and molecular biology. Injury. 38:1115-1130.

53. O'Driscoll, S.W., and Fitzsimmons, J.S. 2001. The role of periosteum in cartilage repair. Clin. Orthop. Relat. Res. 391(Suppl.):S190-S207.

54. Orwoll, E.S. 2003. Toward an expanded understanding of the role of the periosteum in skeletal health. J. Bone Miner. Res. 18:949-954.

55. Zhang, X., et al. 2005. Periosteal progenitor cell fate in segmental cortical bone graft transplantations: implications for functional tissue engineering. J. Bone Miner. Res. 20:2124-2137.

56. Tiyapatanaputi, P., et al. 2004. A novel murine segmental femoral graft model. J. Orthop. Res. 22:1254-1260.

57. Vortkamp, A., et al. 1998. Recapitulation of signals regulating embryonic bone formation during postnatal growth and in fracture repair. Mech. Dev. 71:65-76.

58. Sandell, L.J., and Adler, P. 1999. Developmental patterns of cartilage. Front. Biosci. 4:D731-D742.

59. Nye, H.L., Cameron, J.A., Chernoff, E.A., and Stocum, D.L. 2003. Regeneration of the urodele limb: a review. Dev. Dyn. 226:280-294.

60. Bostrom, M.P., and Camacho, N.P. 1998. Potential role of bone morphogenetic proteins in fracture healing. Clin. Orthop. Relat. Res. 355(Suppl.):S274-S282.

61. Tsuji, K., et al. 2006. BMP2 activity, although dispensable for bone formation, is required for the initiation of fracture healing. Nat. Genet. 38:1424-1429.

62. Molofsky, A.V., Pardal, R., and Morrison, S.J. 2004. Diverse mechanisms regulate stem cell self-renewal. Curr. Opin. Cell Biol. 16:700-707.

63. Lehmann, W., et al. 2005. Tumor necrosis factor alpha (TNF-alpha) coordinately regulates the expression of specific matrix metalloproteinases (MMPS) and angiogenic factors during fracture healing. Bone. 36:300-310.

64. Baldik, Y., et al. 2005. Deletion of iNOS gene impairs mouse fracture healing. Bone. 37:32-36.

65. Ho, M.L., Chang, J.K., and Wang, G.J. 1998. Effects of ketorolac on bone repair: A radiographic study in modeled demineralized bone matrix grafted rabbits. Pharmacology. 57:148-159.

66. Sudmann, E., and Hagen, T. 1976. Indomethacininduced delayed fracture healing. Arch. Orthop. Unfallchir. 85:151-154.

67. Zhang, X., et al. 2002. Cyclooxygenase-2 regulates mesenchymal cell differentiation into the osteoblast lineage and is critically involved in bone repair. J. Clin. Invest. 109:1405-1415.

68. Olmsted-Davis, E., et al. 2007. Hypoxic adipocytes pattern early heterotopic bone formation. Am. J. Pathol. 170:620-632.

69. Mahy, P.R., and Urist, M.R. 1988. Experimental heterotopic bone formation induced by bone morphogenetic protein and recombinant human interleukin-1B. Clin. Orthop. Relat. Res. 237:236-244.

70. Kang, Q., et al. 2004. Characterization of the distinct orthotopic bone-forming activity of 14 BMPs using recombinant adenovirus-mediated gene delivery. Gene Ther. 11:1312-1320.

71. Li, G., et al. 2005. Differential effect of BMP4 on NIH/3T3 and C2C12 cells: implications for endochondral bone formation. J. Bone Miner. Res. 20:1611-1623.

72. Xie, C., et al. 2007. Structural bone allograft combined with genetically engineered mesenchymal stem cells as a novel platform for bone tissue engineering. Tissue Eng. 13:435-445.

73. Kaplan, F.S., et al. 2006. Dysregulation of the BMP-4 signaling pathway in fibrodysplasia ossificans progressiva. Ann. N. Y. Acad. Sci. 1068:54-65.

74. Kan, L., Hu, M., Gomes, W.A., and Kessler, J.A. 2004. Transgenic mice overexpressing BMP4 develop a fibrodysplasia ossificans progressiva (FOP)-like phenotype. Am. J. Pathol. 165:1107-1115.

75. Hannallah, D., et al. 2004. Retroviral delivery of
Noggin inhibits the formation of heterotopic ossification induced by BMP-4, demineralized bone matrix, and trauma in an animal model. J. Bone Joint Surg. Am. 86-A:80-91.

76. Arikawa, T., Omura, K., and Morita, I. 2004. Regulation of bone morphogenetic protein- 2 expression by endogenous prostaglandin E2 in human mesenchymal stem cells. J. Cell. Physiol. 200:400-406.

77. Nakagawa, K., Imai, Y., Ohta, Y., and Takaoka, K. 2007. Prostaglandin E(2) EP4 agonist (ONO-4819) accelerates BMP-induced osteoblastic differentiation. Bone. 41:543-548.

78. Grohs, J.G., Schmidt, M., and Wanivenhaus, A. 2007. Selective COX-2 inhibitor versus indomethacin for the prevention of heterotopic ossification after hip replacement: a double-blind randomized trial of 100 patients with 1-year follow-up. Acta Orthop. 78:95-98.

79. van der Heide, H.J., Rijnberg, W.J., Van Sorge, A., Van Kampen, A., and Schreurs, B.W. 2007. Similar effects of rofecoxib and indomethacin on the incidence of heterotopic ossification after hip arthroplasty. Acta Orthop. 78:90-94.

80. Saudan, M., et al. 2007. Celecoxib versus ibuprofen in the prevention of heterotopic ossification following total hip replacement: a prospective randomised trial. J. Bone Joint Surg. Br. 89:155-159.

81. Shore, E.M., et al. 2006. A recurrent mutation in the BMP type I receptor ACVR1 causes inherited and sporadic fibrodysplasia ossificans progressiva. Nat. Genet. 38:525-527.

82. De La Pena, L.S., et al. 2005. Fibrodysplasia ossificans progressiva (FOP), a disorder of ectopic osteogenesis, misregulates cell surface expression and trafficking of BMPRIA. J. Bone Miner. Res. 20:1168-1176.

83. Deasy, B.M., et al. 2005. Long-term self-renewal of postnatal muscle-derived stem cells. Mol. Biol. Cell. 16:3323-3333.

84. Kuroda, R., et al. 2006. Cartilage repair using bone morphogenetic protein 4 and muscle-derived stem cells. Arthritis Rheum. 54:433-442.

85. Zheng, B., et al. 2007. Prospective identification of myogenic endothelial cells in human skeletal muscle. Nat. Biotechnol. 25:1025-1034.

86. Buckwalter, J.A., Mankin, H.J., and Grodzinsky, A.J. 2005. Articular cartilage and osteoarthritis. Instr. Course Lect. 54:465-480.

87. Goldring, M.B. 2000. The role of the chondrocyte in osteoarthritis. Arthritis Rheum. 43:1916-1926.

88. Aigner, T., Gluckert, K., and von der Mark, K. 1997. Activation of fibrillar collagen synthesis and phenotypic modulation of chondrocytes in early human osteoarthritic cartilage lesions. Osteoarthr. Cartil. 5:183-189.

89. Martel-Pelletier, J., Mineau, F., Jovanovic, D., Di Battista, J.A., and Pelletier, J.P. 1999. Mitogen activated protein kinase and nuclear factor- $\mathrm{\kappa}$ together regulate interleukin-17-induced nitric oxide production in human osteoarthritic chondrocytes: Possible role of transactivating factor mitogenactivated protein kinase-activated protein kinase (MAPKAPK). Arthritis Rheum. 42:2399-2409.

90. Buckwalter, J.A., and Mankin, H.J. 1997. Articular cartilage. J. Bone Joint Surg. 79A:600-632.

91. Smith, G.N., Jr. 2006. The role of collagenolytic matrix metalloproteinases in the loss of articular cartilage in osteoarthritis. Front. Biosci. 11:3081-3095.

92. Aigner, T., Kim, H.A., and Roach, H.I. 2004. Apoptosis in osteoarthritis. Rheum. Dis. Clin. North Am. 30:639-653, xi.

93. Goldring, S.R., and Goldring, M.B. 2004. The role of cytokines in cartilage matrix degeneration in osteoarthritis. Clin. Orthop. Relat Res. 427(Suppl.):S27-S36.

94. Goldring, M.B., and Goldring, S.R. 2007. Osteoarthritis. J. Cell. Physiol. 213:626-634.

95. Goldring, M.B., and Berenbaum, F. 2004. The regulation of chondrocyte function by proinflammatory mediators: prostaglandins and nitric oxide. Clin. Orthop. Relat. Res. 427(Suppl.):S37-S46.

96. Henrotin, Y.E., Bruckner, P., and Pujol, J.P. 2003. The role of reactive oxygen species in homeostasis and degradation of cartilage. Osteoarthr. Cartil. 11:747-755.

97. Henrotin, Y., Kurz, B., and Aigner, T. 2005. Oxygen and reactive oxygen species in cartilage degradation: friends or foes? Osteoarthr. Cartil. 13:643-654.

98. Lo, Y.Y., Conquer, J.A., Grinstein, S., and Cruz, T.F. 1998. Interleukin-1 beta induction of $\mathrm{c}$-fos and collagenase expression in articular chondrocytes: involvement of reactive oxygen species. J. Cell. Biochem. 69:19-29.

99. Clancy, R.M., Gomez, P.F., and Abramson, S.B. 2004. Nitric oxide sustains nuclear factor kappaB activation in cytokine-stimulated chondrocytes. Osteoarthr. Cartil. 12:552-558.

100.Morita, K., et al. 2007. Reactive oxygen species induce chondrocyte hypertrophy in endochondral ossification. J. Exp. Med. 204:1613-1623.

101.Fragonas, E., et al. 1998. Sensitivity of chondrocytes of growing cartilage to reactive oxygen species. Biochim. Biophys. Acta. 1425:103-111.

102.Henrotin, Y., and Kurz, B. 2007. Antioxidant to treat osteoarthritis: dream or reality? Curr. Drug Targets. 8:347-357.

103.Landrigan, P.J. 1991. Current issues in the epidemiology and toxicology of occupational exposure to lead. Toxicol. Ind. Health. 7:9-14.

104.Hicks, D., et al. 1996. Effects of lead on growth plate chondrocyte phenotype. Toxicol. Appl. Pharmacol. 140:164-172.

105.Nemoto, K., et al. 2000. Gene expression of neurotrophins and their receptors in lead nitrate-induced rat liver hyperplasia. Biochem. Biophys. Res. Commun. 275:472-476.

106. Barry, P. 1975. A comparison of concentrations of lead in human tissues. Br. J. Ind. Med. 32:119-139.

107. Pearl, M., and Boxt, L.M. 1980. Radiographic findings in congenital lead poisoning. Radiology. 136:83-84.

108.Schwartz, J., Angle, C.R., Pirkle, J.L., and Pitcher, H. 1986. Relationship between childhood blood-lead levels and stature. Pediatrics. 77:281-288.

109.Shukla, R., et al. 1989. Fetal and infant lead exposure: effects on growth in stature. Pediatrics. 84:604-612.

110.Shukla, R., Dietrich, K.N., Bornschein, R.L., Berger, O.G., and Hammond, P.B. 1991. Lead exposure and growth in the early pre-school child: A follow-up report from the Cincinnati lead study. Pediatrics. 88:886-892.

111.Zuscik, M.J., et al. 2007. Lead induces chondrogenesis and alters transforming growth factorbeta and bone morphogenetic protein signaling in mesenchymal cell populations. Environ. Health Perspect. 115:1276-1282.

112.Zuscik, M.J., et al. 2002. Lead alters parathyroid hormone-related peptide and transforming growth factor-beta 1 effects and AP- 1 and NF-kappaB signaling in chondrocytes. J. Orthop. Res. 20:811-818.

113. Carmouche, J.J., et al. 2005. Lead exposure inhibits fracture healing and is associated with increased chondrogenesis, delay in cartilage mineralization, and a decrease in osteoprogenitor frequency. Environ. Health Perspect. 113:749-755.

114.Liao, X., Lee, G.S., Shimizu, H., and Collins, M.D. 2007. Comparative molecular pathology of cadmium- and all-trans-retinoic acid-induced postaxial forelimb ectrodactyly. Toxicol. Appl. Pharmacol. 225:47-60.

115.Hsieh, S.C., et al. 2006. The inhibition of osteogenesis with human bone marrow mesenchymal stem cells by $\mathrm{CdSe} / \mathrm{ZnS}$ quantum dot labels. Biomaterials. 27:1656-1664.

116.Litchfield, T.M., Ishikawa, Y., Wu, L.N., Wuthier, R.E., and Sauer, G.R. 1998. Effect of metal ions on 


\section{review series}

calcifying growth plate cartilage chondrocytes. Calcif. Tissue Int. 62:341-349.

117.Schmitz, M.A., Finnegan, M., Natarajan, R., and Champine, J. 1999. Effect of smoking on tibial shaft fracture healing. Clin. Orthop. Relat. Res. 365:184-200.

118. Brown, C.W., Orme, T.J., and Richardson, H.D. 1986. The rate of pseudarthrosis (surgical nonunion) in patients who are smokers and patients who are nonsmokers: A comparison study. Spine. 11:942-943.

119.Deguchi, M., Rapoff, A.J., and Zdeblick, T.A. 1998. Posterolateral fusion for isthmic spondylolisthesis in adults: analysis of fusion rate and clinical results. J. Spinal Disord. 11:459-464.

120.Hadley, M.N., and Reddy, S.V. 1997. Smoking and the human vertebral column: A review of the impact of cigarette use on vertebral bone metabolism and spinal fusion. Neurosurgery. 41:116-124.

121.Mooney, V., McDermott, K.L., and Song, J. 1999. Effects of smoking and maturation on long-term maintenance of lumbar spinal fusion success J. Spinal Disord. 12:380-385.

122.Hilibrand, A.S., Fye, M.A., Emery, S.E., Palumbo, M.A., and Bohlman, H.H. 2001. Impact of smoking on the outcome of anterior cervical arthrodesis with interbody or strut-grafting. J. Bone Joint Surg. Am. 83-A:668-673.

123.Blumenthal, S.L., Baker, J., Dossett, A., and Selby, D.K. 1988. The role of anterior lumbar fusion for internal disc disruption. Spine. 13:566-569.

124.Hanley, E.N., Jr., and Levy, J.A. 1989. Surgical treatment of ischemic lumbosacral spondylolisthesis, analysis of variables influencing results. Spine. 14:48-50

125.El-Zawawy, H.B., Gill, C.S., Wright, R.W., and Sandell, L.J. 2006. Smoking delays chondrogenesis in a mouse model of closed tibial fracture healing. J. Orthop. Res. 24:2150-2158. 